\title{
ELEVAÇÃO DA VIDA ÚTIL DOS PNEUS DIANTEIROS DAS CARREGADEIRAS CATERPILLAR MOD:992G/K NA GERÊNCIA DE OPERAÇÕES ÁGUA LIMPA *
}

Daniela Miranda ${ }^{1}$

Fábio José ${ }^{2}$

Amarsirley Silva ${ }^{3}$

\section{Resumo}

No processo produtivo de minério de ferro, dezenas de equipamentos são utilizados no preparo da mina para as operações de perfuração, desmonte, carregamento e transporte do minério retirado das minas a céu aberto. A iniciativa de trabalhar com a elevação da vida útil dos pneus dianteiros da frota de 992G/K (Caterpillar) ocorreu pelo fato do mesmo ser um dos custos mais altos na operação de mina, ser o maior representante de geração de resíduos de borracha e dentre os pneus em operação, a frota de carregadeiras $992 \mathrm{G} / \mathrm{K}$ apresentava performance dos pneus do eixo dianteiro inferior à recomendação do fabricante e ao nosso orçamento anual, por isso se tornou foco do trabalho a ser desenvolvido. A metodologia aplicada para a elaboração do projeto foi Seis Sigma, que possibilitou a análise dos dados e alcance dos resultados acima da meta estabelecida por meio de ações processuais estruturantes e comportamentais, levando à redução da variabilidade.

Palavras-chave: Manutenção; Carregadeira; Pneu; Vida Útil.

\section{USEFULL LIFE INCREASE OF THE FRONT TIRES OF CATERPILLAR LOADERS MOD: 992G/K IN OPERATIONS MANAGEMENT IN ÁGUA LIMPA}

\begin{abstract}
In the iron ore production process, dozens of equipment are used on prepare of the mine for the operations of drilling, dismantle, loading and transportation of the ore withdrawal from open pit mines. The initiative of working with the useful life increase of front tires from the $992 \mathrm{G} / \mathrm{K}$ (Caterpillar) fleet was due to the fact that it is one of the highest costs on mine operation and it is the biggest representative of rubber wastes generation among the tires in operation. The $992 \mathrm{G} / \mathrm{K}$ loader fleet presented front axle tires performance lower than the manufacturer's recommendation and lower than our annual budget, so it became the focus of the work to be developed. The method applied for the project elaboration was Six Sigma, which allowed the data analysis and achievement of results above the established goal through procedural, structuring and behavioral actions, leading to reduction of variability.
\end{abstract}

Keywords: Maintenance; Loader; Tire; Useful life.

1 Bacharel em Administração de Empresas pela Universidade Castelo Branco, MBA Gestão de Projetos pela Rede Doctum, Especialista Green Belt, Analista Operacional PCM - Mina de Água Limpa, VALE S/A, Rio Piracicaba, Minas Gerais, Brasil.

2 Técnico em Mecatrônica pelo Centro Educacional Beldani, Inspetor de Man. de Pneus PCM da Mina de Água Limpa, VALE S/A, Rio Piracicaba, Minas Gerais, Brasil.

3 Bacharel em Administração de Empresas pela Universidade Norte do Paraná, Graduando em Engenharia Elétrica pela Doctum, Especialista Green Belt, Supervisor de Manutenção de equipamentos de carga / terraplanagem, VALE S/A, Rio Piracicaba, Minas Gerais, Brasil. S/A. 


\section{INTRODUÇÃO}

As Minas Centrais ficam localizadas nas cidades de São Gonçalo do Rio Abaixo, Barão de Cocais, Santa Bárbara e Rio Piracicaba. Compõe as Minas Centrais: Água Limpa, Brucutu e Gongo Soco (atualmente paralisada por fim das licenças operacionais). Brucutu se destaca por ser a maior mina do mundo em capacidade inicial de produção de minério de ferro e a $2^{-a}$ maior do Brasil, ficando atrás apenas de Carajás, também da Vale.

Com produção anual de mais de 4 milhões de toneladas de minério de ferro, a Mina de Água Limpa conta com uma frota de 61 equipamentos que são divididos em equipamentos de carga, desmonte, transporte e terraplanagem. Identificado dentre os pneus em operação que a performance dos pneus que operam no eixo dianteiro das carregadeiras $992 \mathrm{G} / \mathrm{K}$ apresentava-se uma performace abaixo da recomendação do fabricante e do nosso orçamento anual, limitando-se assim este trabalho com este foco estabelecido.

\subsection{Pá carregadeira 992G/K}

A Caterpillar apresentou a 992 pela primeira vez em 1968. Essa grande pácarregadeira de rodas tem sido líder global desde então, construindo uma reputação como pá-carregadeira de face segura de operar, produtiva e durável. [1]

Com as configurações de levantamento padrão e alto, essa máquina é compatível com frotas de caminhões 775, 777 e 785 . O equipammento possui 4 pneus, sendo 02 no eixo dianteiro e 02 no eixo traseiro. (Figura 1). A frota de equipamentos deste modelo em operação em Água Limpa é de 4 máquinas que operam em turno initerrupto de $6 \mathrm{~h}$ totalizando em média $18 \mathrm{~h}$ trabalhadas dia.

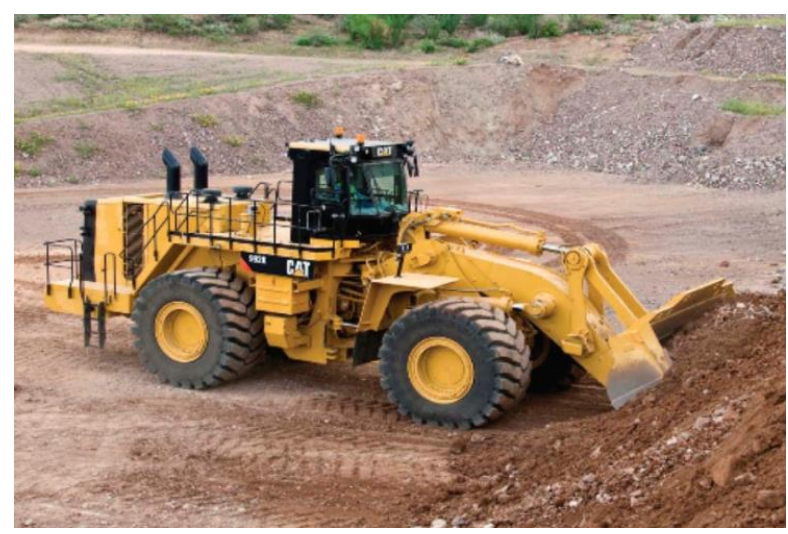

Figura 1. Carregadeira Caterpillar 992G.

\subsection{Pneu Michelin 45/65R45}

Pneu é um artefato circular feito de borracha, tendo como principais funções: transportar, guiar, transmitir, amortecer, rodar e durar.Nas carregadeiras em operação, é utilizado pneu do fabricante Michelin/Bridgestone, com dimensão 45/65R45 ( os numeros indicam a dimensão do pneu e o R indicada a construção do pneu, nesse caso o R é de Radial), desenho XLDD1 L4R, composto A. (Figura 2) Possui uma escultura muito recortada, aberta no centro e agressiva, que dá aos pneus excelentes resultados em tração, mais aderência transversal e mais eficácia ao 
carregar. A velocidade de trabalho máxima é de $14 \mathrm{~km} / \mathrm{h}$ e a profundidade é de $71 \mathrm{~mm}$ (essa medida pode sofrer variação dependendo do fabricante do pneu)

Sua durabilidade depende basicamente de fatores tais como: condições das praças de carga e descarga, pressão de trabalho e modo de operação.

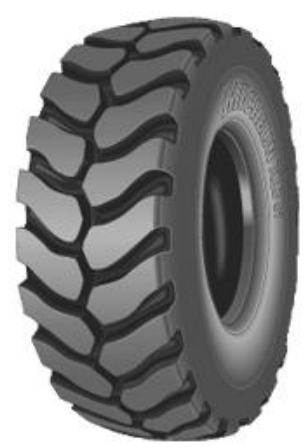

Figura 2. Modelo de Pneu Michelin 45/65R45.

Dimensões:

- Diâmetro da roda: $45^{\prime \prime}$

- Largura nominal: $1.143 \mathrm{~mm}$

- A altura do flanco (lateral) do pneu: $742,95 \mathrm{~mm}$

- O diâmetro total do pneu: $2733 \mathrm{~mm}$

\section{MATERIAIS E MÉTODOS}

Este trabalho baseia-se em análise histórica dos pneus, acompanhamento operacional, observações e análise de variância (ANOVA) cadenciada pela aplicação do trabalho de Seis Sigma, método sistemático e organizado para melhoria de processos.

\subsection{Identificação do problema}

Analisando o histórico de dados de trocas de pneus (dianteiros), percebemos que estamos 14\% abaixo da recomendação do fabricante (3.000 h) (Figura 3)

Figura 3. Período de análise dos dados (2ํㅜㄴimestre de 2013 a $2^{\circ}$ Trimestre de 2015)

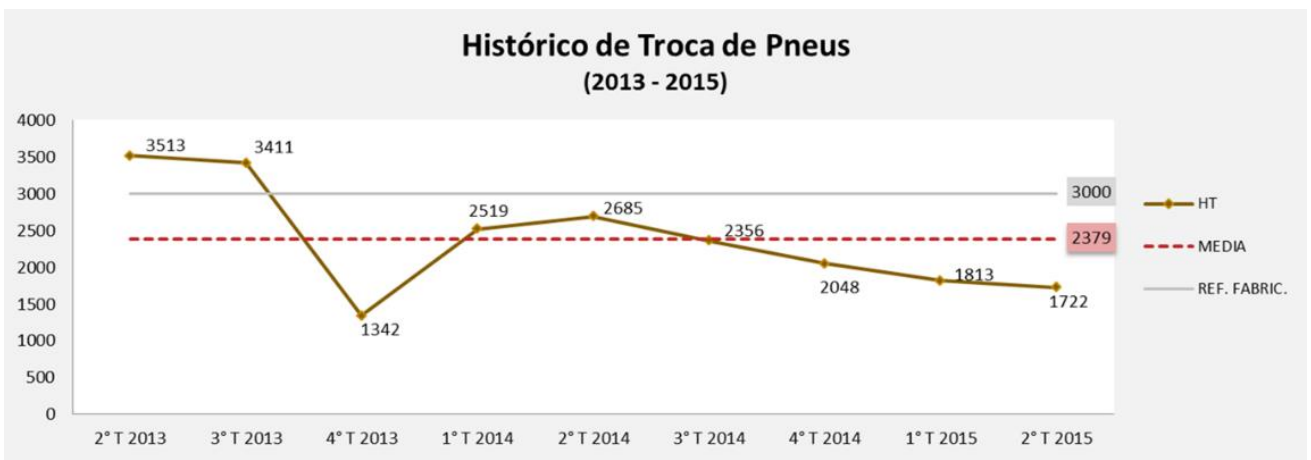

O indicador que será tratado é o Horímetro Final do Pneu menos o Horímetro de instalação (início de operação do pneu), ou seja, a vida útil do pneu. 
Para definição da meta foi utilizado o terceiro quartil, que é o valor que se encontra $75 \%$ da amostra, nesse caso representa $3.382 \mathrm{~h}$, superando o recomendado pelo fabricante de $3000 \mathrm{~h}$ e validada pelo sponsor do projeto. (Figura 4)

Figura 4. Gráfico de probabilidade para definição da meta (utilizado 3ำ quartil)

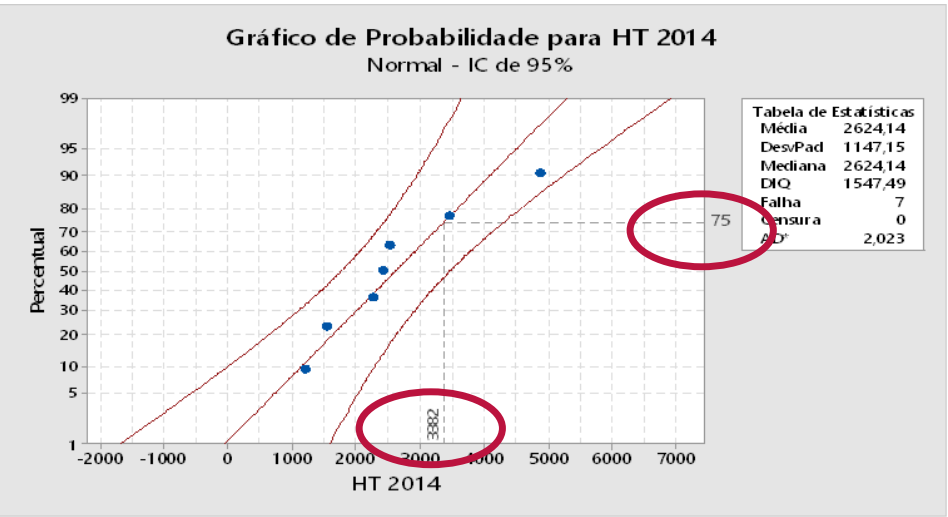

Após estabelecida a meta foi feito uma análise de desempenho do processo para verificar o percentual de atendimento dentro do especificado para o cenário atual, ou seja, dentro da meta proposta. $O$ resultado do teste de normalidade foi $P=1$, ou seja, os dados são normais (Figura 4) o que permite que seja feito a análise de desempenho do processo pelo gráfico de capabilidade tendo como resultado $75 \%$ fora do especificado. (Figura 5)

Figura 4. Teste de normalidade

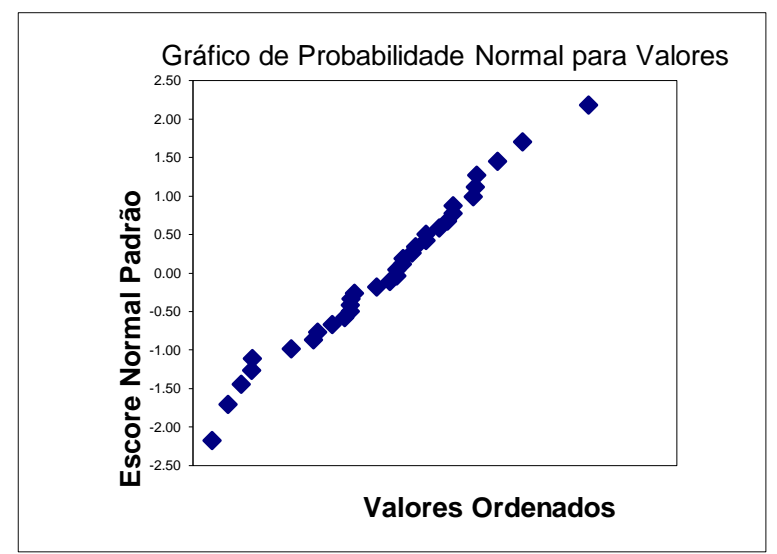

Figura 5. Gráfico de Capabilidade

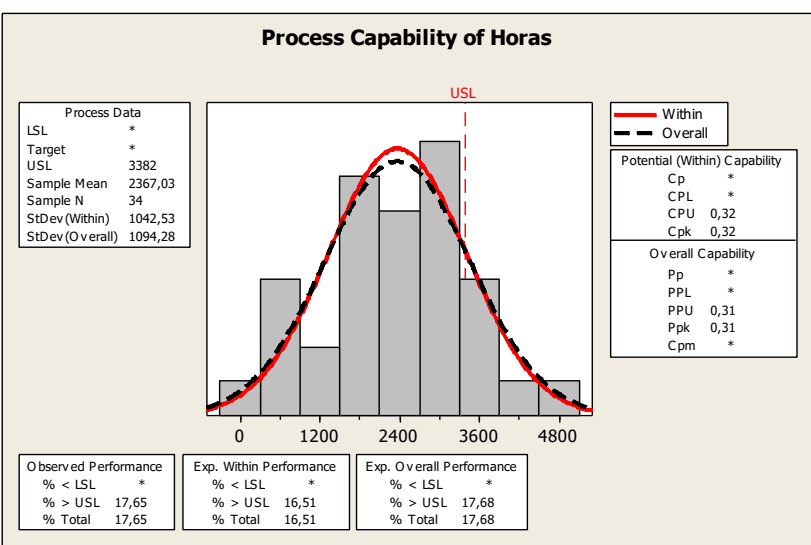

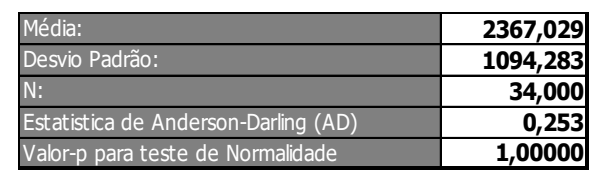

\subsection{Análise do Fenômeno}

Nesta etapa foi realizado estratificação no nível do equipamento, entendendo que a posição em que o pneu opera (lado direito ou esquerdo) não influencia, assim como o local de operação, uma vez que o equipamento não possui frentes de operação fixas, havendo variação do local em regime de rodízio.

Definimos como foco a ser trabalhado: as horas trabalhadas e o desgaste da banda de rodagem dos pneus. 
Foram definidas duas metas específicas para sustentar o desafio proposto: horas trabalhadas e desgaste da banda de rodagem dos pneus.

Onde que para a meta específica de horas trabalhadas, utilizamos o método da Lacuna, considerando os melhores valores de troca de cada pneu do equipamento. $\mathrm{E}$ para a meta específica de desgaste foi utilizada a média do valor $(\mathrm{mm})$ de desgaste (taxa de desgaste) dos pneus com melhor performance histórica, criando-se assim uma referência para a retirada dos pneus do eixo dianteiro. $O$ valor estabelecido foi de $17,1 \mathrm{~mm}$.

\subsection{Análise do Processo}

Utilizado ferramenta brainstorming (Figura 6) para levantamento das causas potenciais foram levantadas 17 causas. Através diagrama de Ishikawa foi possível a relação entre a causa e o efeito do problema.

As causas foram priorizadas utilizando o FMEA (failure mode and effect analysis) [2], que permitiu a análise dos modos de falha e seus efeitos, permitindo relacionar as causas com os controles atuais e realizar a priorização seguindo os critérios de severidade, frequência e detecção. (Figura 7)

Figura 6. Brainstorming: Causas potenciais

\begin{tabular}{|l|l|}
\hline \multicolumn{2}{|c|}{ BRAINSTORMING } \\
\hline \multicolumn{2}{|c|}{ CAUSAS POTENCIAIS } \\
\hline 1 & $\begin{array}{l}\text { Agressões de objetos pontiagudos ou cortantes, que deixam marcas visíveis no } \\
\text { local danificado }\end{array}$ \\
\hline 2 & Geração de bolhas na lateral do pneu \\
\hline 3 & Separação entre a borracha e a carcaça \\
\hline 4 & Insuficiencia de pressão \\
\hline 5 & Velocidades elevadas \\
\hline 6 & Sobrecarga \\
\hline 7 & Arraste do pneu sobre o solo \\
\hline 8 & Esterçar o equipamento parado \\
\hline 9 & Excesso / insuficiência de pressão \\
\hline 10 & Construção do pneu \\
\hline 11 & Load and Carry \\
\hline 12 & Aquecimento da roda \\
\hline 13 & Execesso de torque \\
\hline 14 & Corte \\
\hline 15 & Impactos contra obstáculos cortantes \\
\hline 16 & Martelamento excessivo do pneu \\
\hline 17 & Contato com combustíveis, lubrificantes, óleo queimado, graxas, etc. \\
\hline
\end{tabular}

Figura 7. FMEA

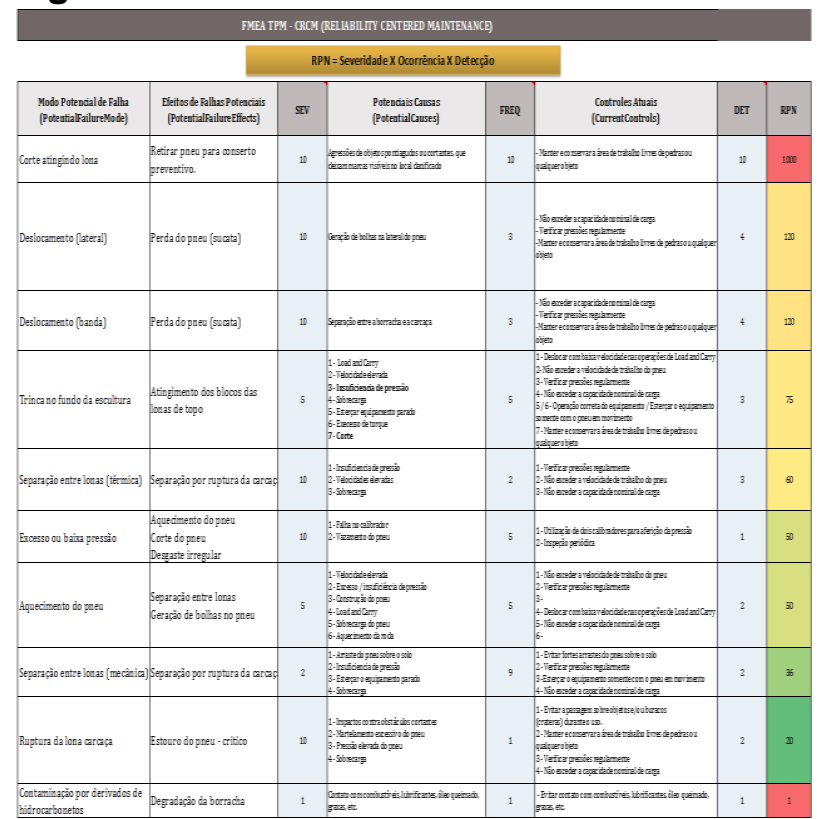

Foram levantadas 5 causas potenciais, sendo elas:

- Agressões de objetos pontiagudos ou cortantes, que deixam marcas visíveis no local danificado;

- Geração de bolhas na lateral do pneu;

- Separação entre a borracha e a carcaça;

- Martelamento excessivo do pneu;

- Excesso ou insuficiência de pressão.

Para comprovar as causas priorizadas foi realizado a quantificação dos danos ocorridos nos pneus e levantamento fotográfico, comprovando as quatro primeiras causas mencionadas anteriormente. 
Para comprovação da causa levantada: excesso ou insuficiência de pressão, foi realizado uma pesquisa de Benchmark, afim de verificar qual operação similar, apresentava resultados de vida útil acima da recomendação do fabricante, e comparar então com a pressão de trabalho utilizado nos pneus em operação.

Identificamos uma operação similar na empresa Samarco, município de Mariana, onde a pressão praticada estava acima da usada nos processos de Água Limpa que seguia a recomendação do fabricante conforme manual, 94PSI. (Figura 8)

Figura 8. TECHNICAL DATA - EARTHMOVER tyres - 2012 EDITION [3]

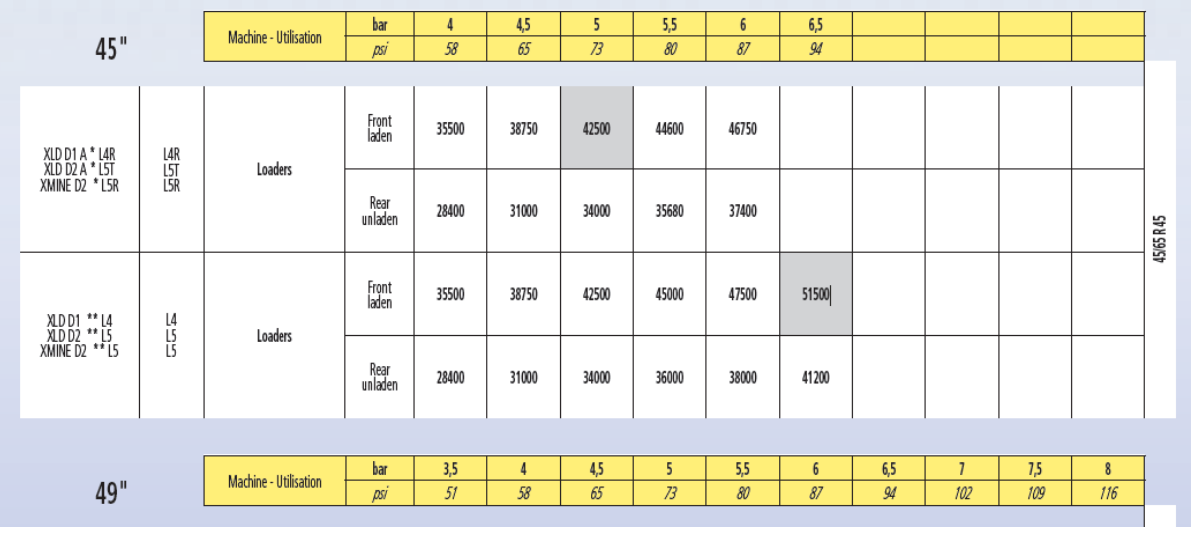

Considerando essa diferença de pressão praticada, iniciamos um teste para verificar qual pressão ideal para os pneus. Avaliamos por um período as pressões de 107 e 114 PSI.

Optamos pela análise de variância [4], também chamada de ANOVA, utilizada para comprovar a diferença entre médias de duas ou mais condições do processo. $O$ primeiro passo foi a realização do teste de LEVENE, que testa duas hipóteses para verificar a variabilidade do processo. O resultado do teste apresentou resultado de 0,098 e concluímos que não existe diferenças entre as variabilidades. (Figura 9)

Figura 9. Teste de Levene

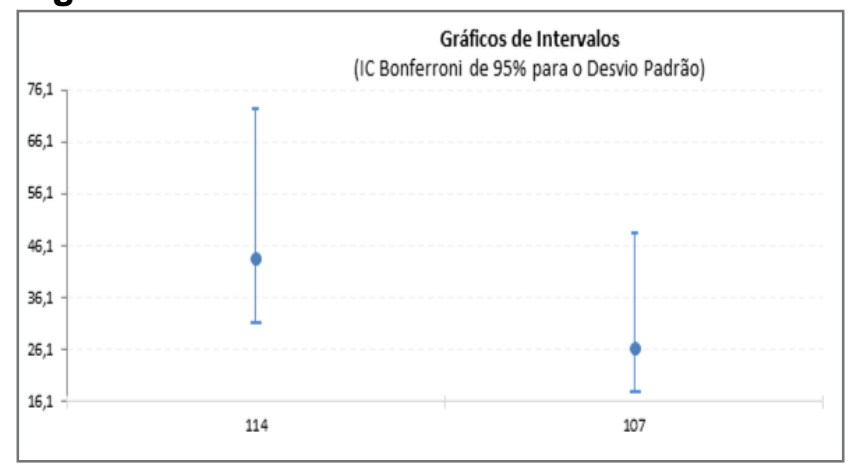

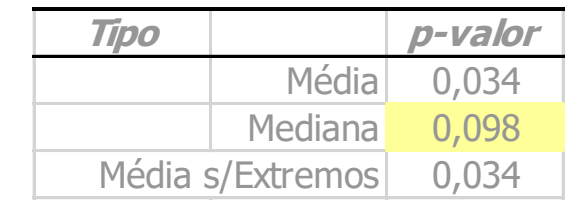

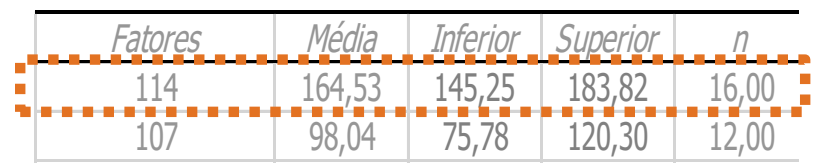

Com este resultado favorável foi possível dar continuidade no teste e realizar a análise de variância (comparação entre as médias), após os testes das duas hipóteses, 0 resultado foi 0 , ou seja, pelo menos um processo apresenta média diferente dos demais. Indicando um melhor resultado como 114 PSI. Comprovando-se assim a causa relacionada à pressão.

Com esse teste foi possível determinar uma nova faixa de pressão de trabalho para os pneus em operação. 


\subsection{Estabelecimento do plano de ação}

Para estabelecimento do plano de ação, usamos a ferramenta Brainstorming e realizamos o levantamento das possíveis soluções, relacionando com as causas comprovadas. Essas ações foram priorizadas obedecendo o seguinte critério: Complexidade, custo, impacto sobre a causa e prazo. Estabelecemos 20 ações conforme figura 10.

Figura 10. Plano de ação priorizado

\begin{tabular}{|c|c|c|c|c|c|}
\hline CAUSA & $\begin{array}{l}\text { O qué } \\
\text { Medida (0 qué tazer?) }\end{array}$ & $\begin{array}{l}\text { Quvem? } \\
\text { Quem irá lazer? }\end{array}$ & $\begin{array}{c}\text { Quando? } \\
\text { Quando sera letito? }\end{array}$ & $\begin{array}{c}\text { Como? } \\
\text { Como será felito? }\end{array}$ & $\begin{array}{c}\text { Onde? } \\
\text { Onde será feito? }\end{array}$ \\
\hline \multirow{5}{*}{ 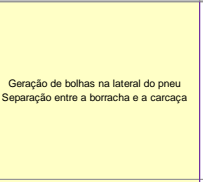 } & \multirow{5}{*}{ Instalar balança de pesagem de equipamentos de mina. } & Fábio José & 030042015 & $\begin{array}{l}\text { Solictiar Michelin a disponiblididade da balança na } \\
\text { Mina de Agua Limpa }\end{array}$ & $\mathrm{PCM}$ \\
\hline & & Fábio José & 0610422015 & \begin{tabular}{|l|} 
Transportar balanca de Mariana para Agua Limpa \\
\end{tabular} & COMPLEXO MARIIANA \\
\hline & & Fábio José & 28:04:2015 & Instalaçăo da balança & $\mathrm{PCM}$ \\
\hline & & Fábio José & 040522015 & Testes de pesaagem & $\mathrm{PCM}$ \\
\hline & & Fábio Jose & 1906:2015 & $\begin{array}{l}\text { Anallsar resultadod da pesagem reallzada nas } \\
\text { carregaderias }\end{array}$ & $\mathrm{PCM}$ \\
\hline \multirow{2}{*}{ Excesso / insulicieincia de pressắo } & \multirow{2}{*}{ 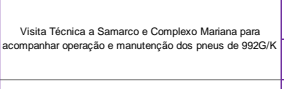 } & Amarsiney & 150522015 & $\begin{array}{l}\text { Agendar vista com oresponsável de manutençắo } \\
\text { de pneus na empresa Samarco / Mina de Alegria }\end{array}$ & $\mathrm{PCM}$ \\
\hline & & $\begin{array}{l}\text { Daniela Miranda } \\
\text { Fabio oss } \\
\text { Jario Marins }\end{array}$ & 2905:2015 & Realizar vista tecrica & SAMAACO \\
\hline \multirow{2}{*}{ Excesso / insuluciéncia de pressáa } & \multirow{2}{*}{ 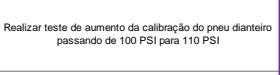 } & Fábio & 2705/2015 & Definir equipamento de teste & $\mathrm{POM}$ \\
\hline & & Cistiane & 020662015 & 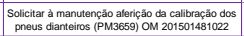 & OFIIINA EQTOS MÓVEIS \\
\hline \multirow{2}{*}{ 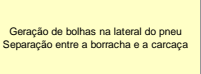 } & \multirow{2}{*}{ 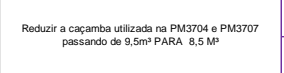 } & Glaukon Alves & 020622015 & 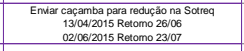 & $\mathrm{POM}$ \\
\hline & & Juliana & 31077/2015 & Aplicar cacambar retomada no equipamento & OFIIINA EQTOS MÓvEIS \\
\hline $\begin{array}{l}\text { Agress sees de de bietos pontiagudos ou } \\
\text { conanantes, que dexixam marcas usisiots no } \\
\text { local danificado }\end{array}$ & $\begin{array}{l}\text { Limitar a operaçăo da PM3704 e PM3707 na alimentąăăo } \\
\text { da usina e baia }\end{array}$ & Junio Oliverira & 2906522015 & 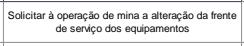 & $\mathrm{PCM}$ \\
\hline Excesso/ insuliciéncia de pressåa & Teste no pneu da PM3704 & Fábio & 101077/2015 & 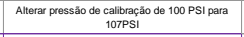 & $\mathrm{PCM}$ \\
\hline \multirow{2}{*}{$\begin{array}{l}\text { Agress seses de bijess pontiagudos ou } \\
\text { contantes, que dexam marcas usisieis no } \\
\text { local danificado }\end{array}$} & \multirow{2}{*}{ 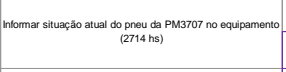 } & Daniela & 1707/2015 & Elaborar intormativo & $\mathrm{PCM}$ \\
\hline & & Fabbio & 220772015 & $\begin{array}{l}\text { Fixar no equipamento/ / Realizar dilalogo } \\
\text { compontanentalal com o operador }\end{array}$ & MINA \\
\hline 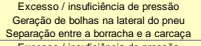 & $\begin{array}{l}\begin{array}{l}\text { Acompanhar os pneus da PM3707 atrawes do sott TPMS } \\
\text { (Mems) }\end{array} \\
\end{array}$ & Fábio & $0306 / 2015$ & \begin{tabular}{|c|} 
Untilizar aparalho PDA que registra temperatura \\
pressáo dos pneus em tempor peal
\end{tabular} & MNA \\
\hline 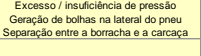 & 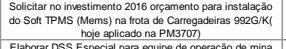 & Daniela & 2509/2015 & $\begin{array}{c}\text { Enviar solichlaçăo com especilicąăa para Gilmara } \\
\text { / Tassia }\end{array}$ & $\mathrm{PCM}$ \\
\hline \multirow{2}{*}{ 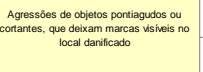 } & 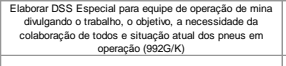 & Daniela & 07/08/2015 & 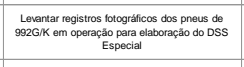 & $\mathrm{PCM}$ \\
\hline & Divilgar a equipe de operacago de de mina o DSS Especial & Flavano & 14082015 & Divilgar em DSS e registrar em OJT & MNA \\
\hline 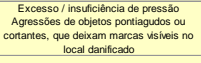 & 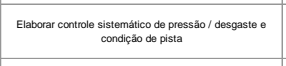 & Daniela & 29065/2015 & 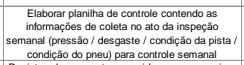 & $\mathrm{PCM}$ \\
\hline \multirow{2}{*}{ 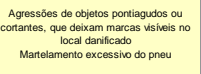 } & $\begin{array}{l}\text { Fazer analise de incidente de toda acour } \\
\text { cone de preu }\end{array}$ & pres de Operaçádo & 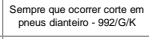 & 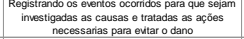 & MINA \\
\hline & 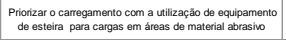 & Superisoreses de Operagáa de Mina & 15/10/2015 & 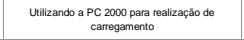 & MINA \\
\hline
\end{tabular}

\subsection{Implantação do plano de ação}

Um cronograma detalhado de acompanhamento da execução das ações garante a efetividade das medidas necessárias. Através de reuniões semanais, a evolução de execução das ações foi acompanhada, concluindo todas dentro dos prazos estabelecidos.

Podemos destacar ações estruturantes como:

- Instalação de balança de pesagem: Utilizado para verificar sobrecarga nos equipamento. (Figura 11)

- Redução de 02 caçambas de $9,5 \mathrm{~m}^{3}$ para $8,5 \mathrm{~m}^{3}$ (Figura 12)

- Utilização do aparelho PDA que permite acompanhar a pressão e temperatura do pneu. (Figura 13) 
Figura 11. Balança pesagem

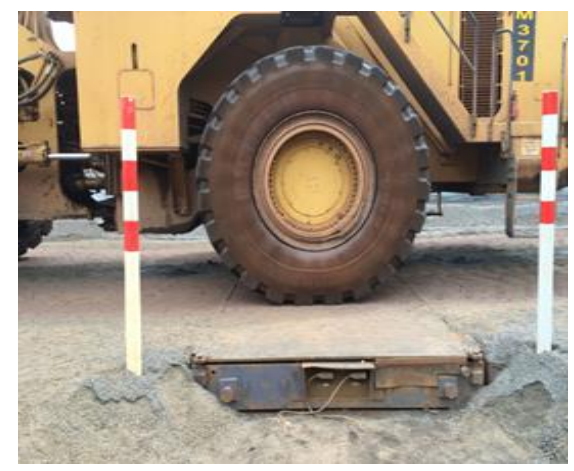

Figura 12. Redução caçamba

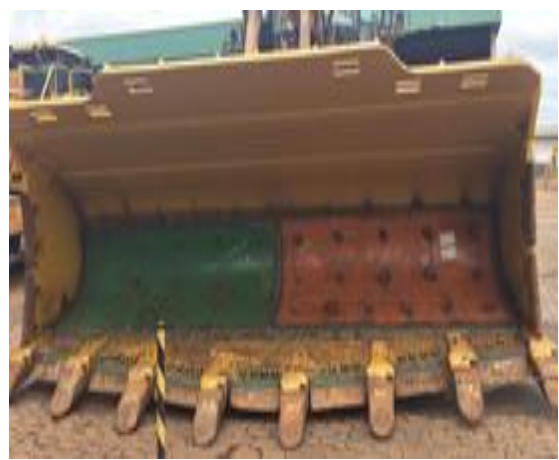

Figura 13. PDA

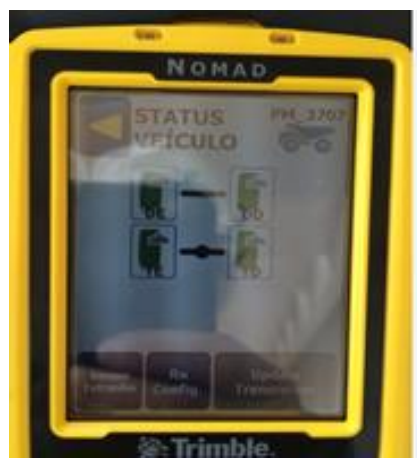

Algumas ações processuais foram importantes para melhora no processo como a adequação dos controles de inspeção / pneu e praça de trabalho. Permitindo um monitoramento sistemático e que atenda às necessidades do processo. Já citado anteriormente, foi alterado a calibração de pressão dos pneus para conforme o resultado do teste realizado (114 PSI)

Além disso, com resultados positivos tivemos ações voltadas ao comportamento dos operadores, entendendo que este é um fator que se destacou nas análises realizadas. O uso de ferramentas pró ativas como diálogos comportamentais, DSS especiais e a simples atitude de marcar em vermelho o comando dos pneus que está em operação, mas que apresentam algum tipo de dano, fez com que o operador se sentisse peça fundamental no projeto. Intuitivamente foi adotada uma operação com atenção redobrada permitindo o envolvimento de todos e fazendo com que o sentimento de dono motivasse os operadores a melhorar as condições operacionais. (Figura 14)

Figura 14. Marcação visual do comando final (componente mecânico) do pneu em operação que apresenta algum dano (corte / trinca) e que está sendo monitorado.

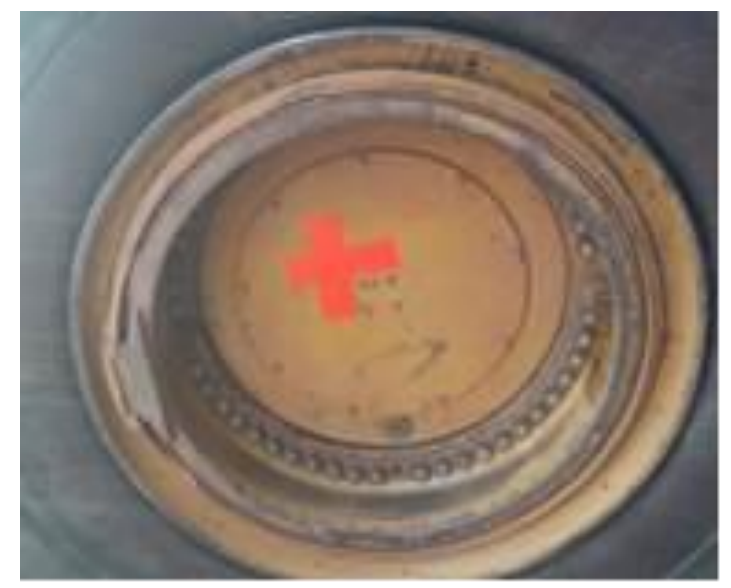

Sem dúvida o conjunto das ações estruturantes, processuais e comportamentais garantiu que bons resultados fossem percebidos.

\section{RESULTADOS E DISCUSSÃO}

\subsection{Verificação dos resultados}


Após a implantação das 20 ações priorizadas, e executadas até novembro de 2015, obteve-se o resultado conforme apresentado na Figura 15. Observa-se que o resultado é solido, e se comprovou em um período acima da recomendação da metodologia Seis Sigma que indica um período de medição de 3 meses.

$\mathrm{O}$ resultado no período de verificação foi de $3.430 \mathrm{~h}$ para uma meta de $3.382 \mathrm{~h}$. As metas especificas estabelecidas também foram superadas o que sustentou este resultado.

O projeto foi medido de set-15 a mar-16 (7 meses), onde os valores permaneceram acima da meta estabelecida.

Figura 14. Boxplot de comparação dos resultados

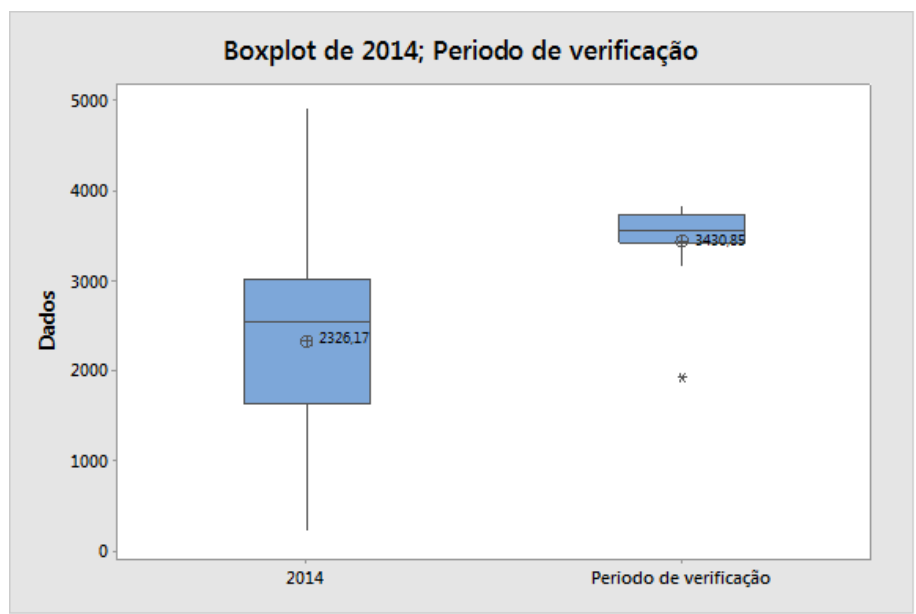

Pela análise de desempenho podemos verificar o deslocamento da média e a diminuição da variabilidade. Tornando o processo mais estável.

Pela comparação da capacidade percebemos que o desvio padrão foi significativamente reduzido, acompanhado do deslocamento da média para dentro do limite de especificação conforme Figura 15.

Figura 15. Comparação da capacidade (Antes / Depois)

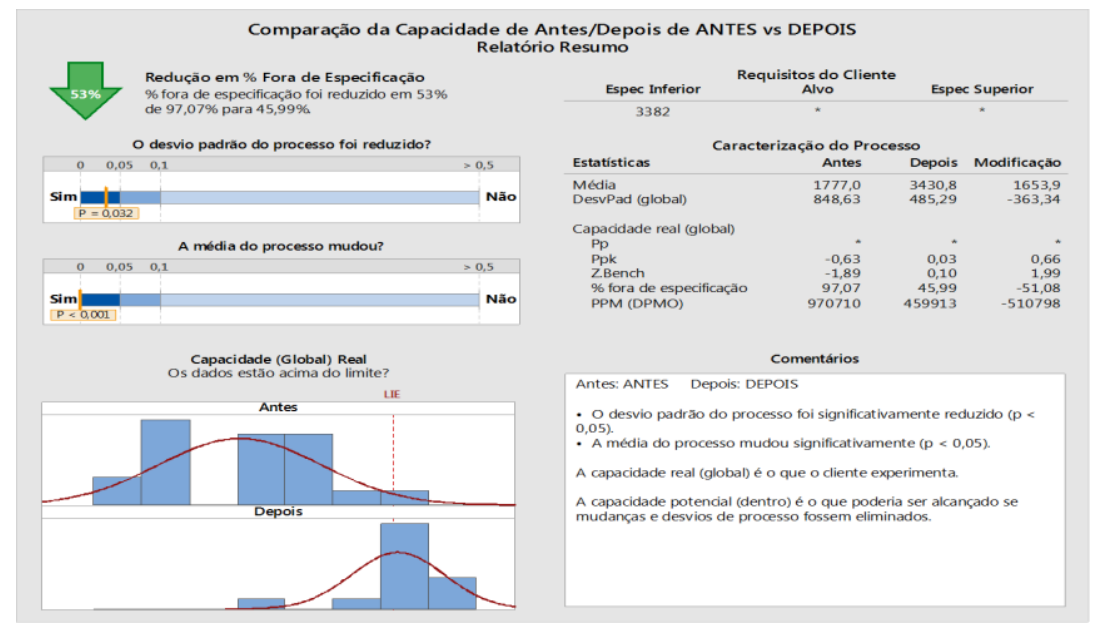

Pela carta I-AM, percebemos a ocorrência de uma causa especial. Onde precisamos investigar. (Figura 16.) 
Figura 16. Carta I-AM

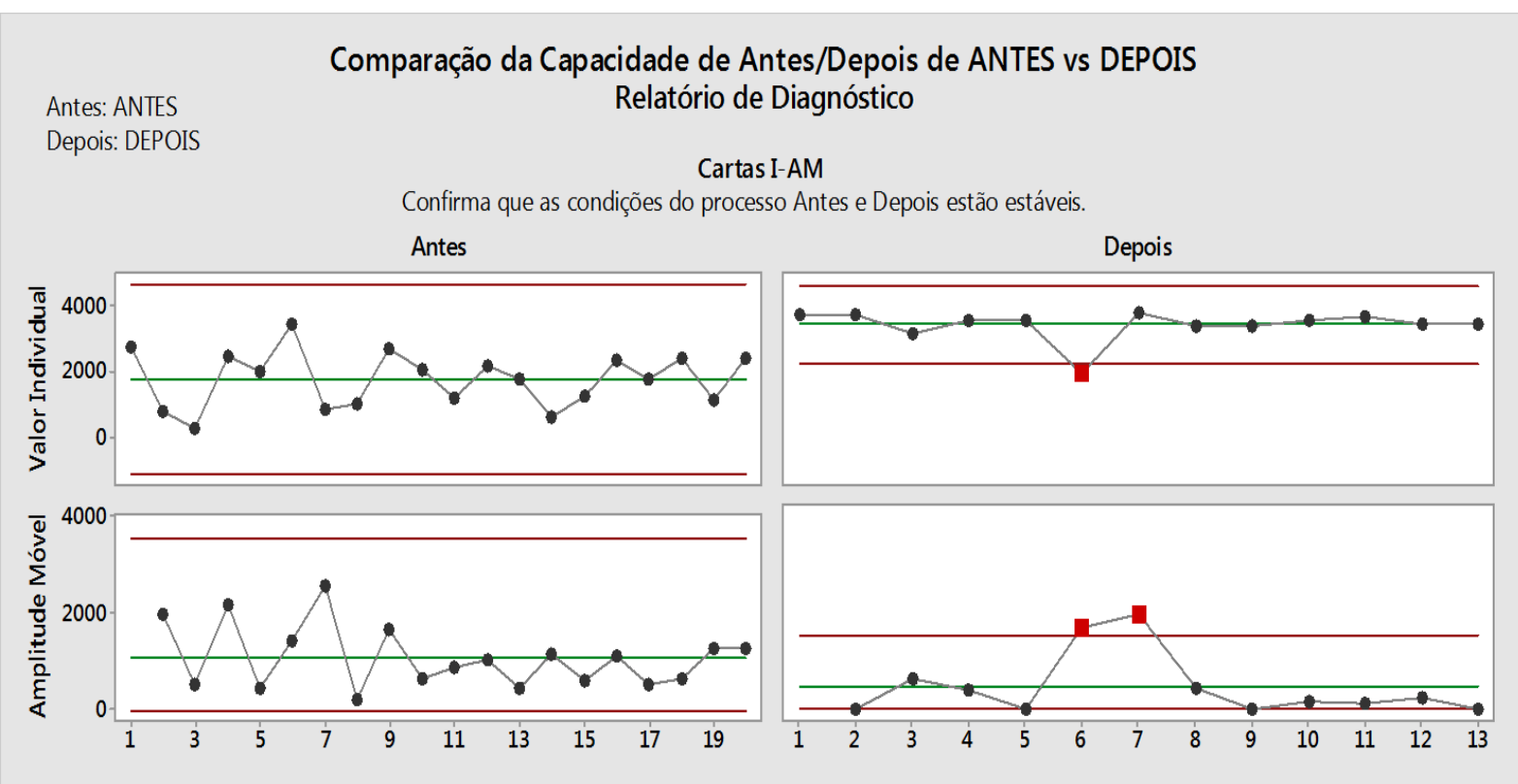

A partir desta ocorrência foi realizado o teste T para uma amostra para comprovar se o valor alcançado deste pneu (de vida precoce) é igual ou diferente do comportamento da família. (Figura 17.) Como o valor de P é igual a 0 , o comportamento do pneu não é igual ao comportamento da família.

Figura 17(a). Gráfico de CEP

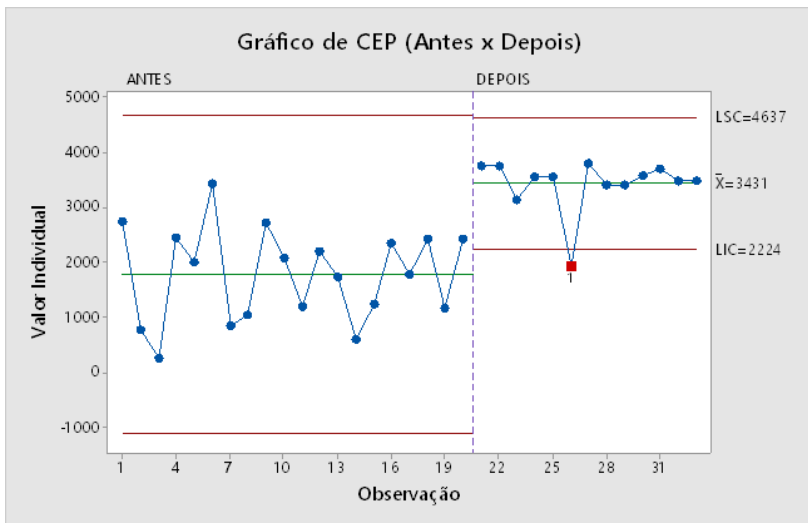

Figura 17 (b). Gráfico de valores individuais

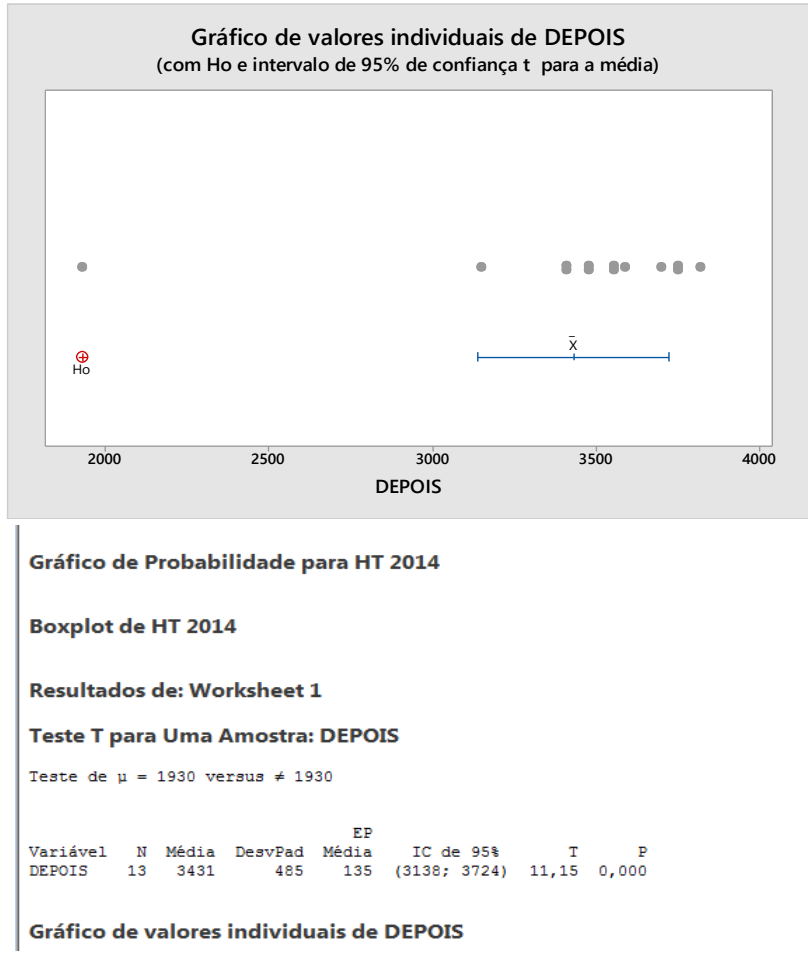

Esta ocorrência foi tratada por uma análise de quase acidente (Figura 18.), por uma equipe multidisciplinar em novembro de 2015. O equipamento operava em um local que não oferecia boas condições com presença de pedras no local. Definido ações a 
partir dessa ocorrência para que não volte a ocorrer, mesmo que este fato isolado não tenha afetado o resultado do projeto.

Figura 18. Análise de quase acidente

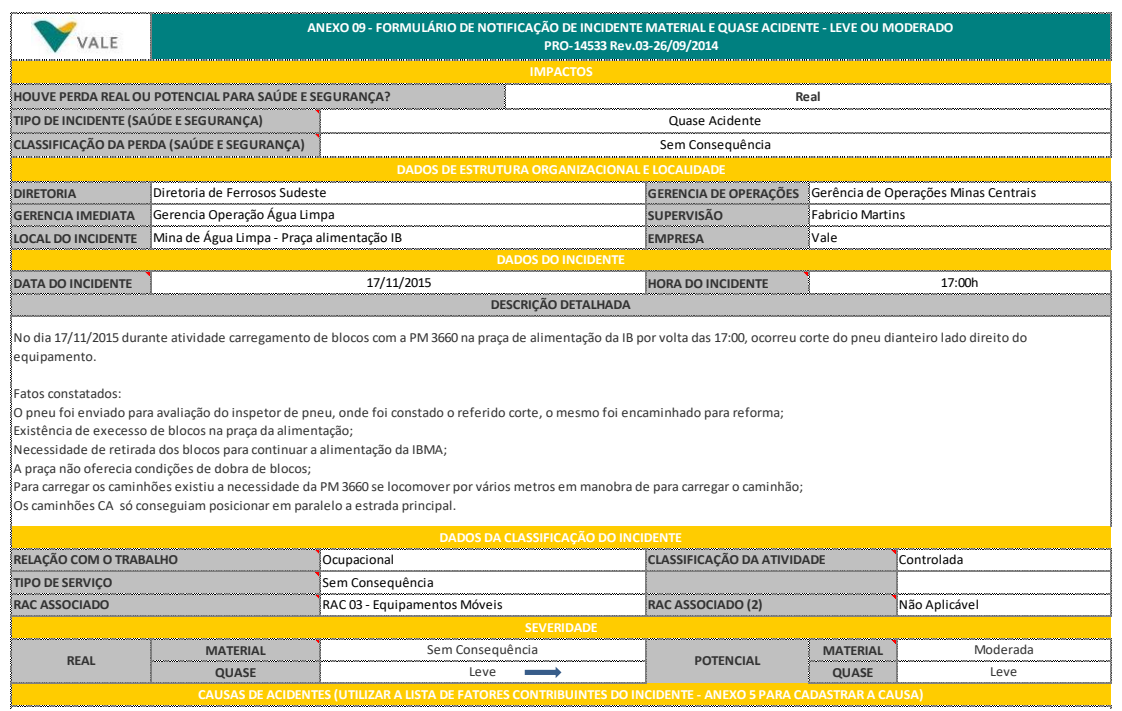

\subsection{Padronização e manutenção dos resultados}

$\mathrm{Na}$ fase de padronização foram desenvolvidos novos indicadores para acompanhamento como as Trocas $(\mathrm{ht}) \mathrm{X}$ Desgaste $(\mathrm{mm})$ e quantificação dos danos por trimestre. Foram revisados os procedimentos operacionais. Elaborado procedimento de inspeção com definição de frequência de inspeção, check de itens relevantes e acompanhamento da pressão dos pneus que deve ser feito sistematicamente.

Para tratar as ações corretivas do processo foi elaborado o OCAP (Figura 19.), que serve de auxílio para a tomada de decisão. A partir do acompanhamento das trocas de pneu, qualquer ocorrência abaixo da vida esperada, deve seguir o fluxo definido, e registrado as alterações no processo por meio do diário de bordo.

Figura 19. OCAP

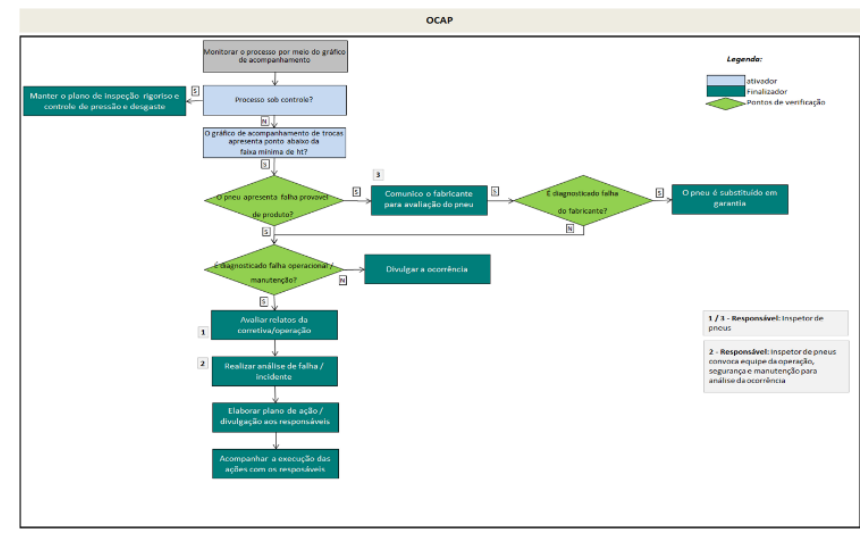




\section{CONCLUSÃO}

O projeto de elevação da vida útil dos pneus dianteiros da frota de carregadeiras Caterpillar $992 \mathrm{G} / \mathrm{K}$ apresentou ganhos significativos nas metas especificas, e na meta geral, saindo de uma média histórica de $2.624 \mathrm{~h}$ para $3.382 \mathrm{~h}$, superando a recomendação do fabricante de $3.000 \mathrm{~h}$ de operação do pneu no eixo dianteiro e atendendo a nossa meta orçamentária. Este ganho em horas trabalhadas, reflete diretamente em economia no custo da área, evitando um gasto de $R \$ 352.784,44$.

A estabilidade dos resultados, a melhoria dos procedimentos internos e o conhecimento das variáveis críticas do processo foram resultados que só poderiam ser alcançados com o comprometimento e engajamento da equipe. Para o sucesso de qualquer projeto, é necessário definir novas formas de trabalho e a aplicação correta da metodologia Seis Sigma permite a obtenção de resultados de forma planejada e clara.

\section{Agradecimentos}

Agradeço o sponsor André Carmo (Gerente de Operações) pelo incentivo e aprovação, à equipe da Manutenção da Mina de Água Limpa, ao supervisor Gener Pena, em especial ao inspetor de pneus, Fábio José, e à controladora/programadora Cristiane Gonçalves pelo apoio técnico, dedicação e disponibilidade de dados. Ao Luiz Gustavo da empresa Seta Desenvolvimento Gerencial pela consultoria metodológica e ao Gestor de Execução, Amarsirley Silva por acreditar e acompanhar o projeto em todas as fases, estimulando o progresso dos resultados.

\section{REFERÊNCIAS}

1 Caterpillar @ 2017 [acesso em 14 jun. Disponível em: http://www.cat.com/pt BR/products/new/equipment/wheel-loaders/large-wheelloaders/1000028683.html

2 Helman, H. Andery P.R.P. (1995). Análise de Falhas (Aplicação dos Métodos FMEA e FTA). Belo Horizonte: Fundação Christiano Ottoni, Escola de Engenharia da UFMG

3 TECHNICAL DATA - EARTHMOVER tyres - 2012 EDITION

4 Aguiar S., Drumond F.B., Werkema, M.C.C. (1196). Análise de Variância: Comparação de várias situações. Belo Horizonte: Fundação Christiano Ottoni, Escola de Engenharia da UFMG. 